\title{
Coarctação da aorta: resultados da cirurgia e análise crítica de diversas técnicas
}

\author{
Carlos R. MORAES*, Jorge V. RODRIGUES*, Cládio A. GOMES**, Euclides TENÓRIO**, Fernando MORAES \\ NETO**, Cleusa Lapa SANTOS**, Ivan de Lima CAVALCANTI**
}

RBCCV 44205-89

\begin{abstract}
MORAES, C. R.; RODRigues, J. V.; GOMES, C. A.; TENÓRIO, E.; MORAES NETO, F.; SANTOS, C. L.; CAVALCANTI, I. L. - Coarctaçăo da aorta: resultados da cirurgia e análise crítica de diversas técnicas. Rev. Bras. Cir. Cardiovasc., 4(2): 168-172, 1989.

RESUMO: Setenta pacientes com idade variável de 14 dias a 49 anos (média 7,6 anos) foram submetidos à correção cirúrgica da coarctação da aorta. Vinte e seis $(37,1 \%)$ estavam no primeiro ano de vida. As técnicas cirúrgicas utilizadas incluíram aortoplastia com enxerto em 30 casos, aortoplastia com subclávia em 28, anastomose término-terminal e nove, interposiçāo de enxerto tubular de Dacron em dois e aortoplastia com subclávia e preservação da circulação para o membro superior esquerdo em um. Ocorreram seis $(8,5 \%)$ óbitos imediatos e dois $(2,8 \%)$ tardios, não relacionados com o tipo de reparo. A mortalidade imediata teve relaçăo direta com a idade, anomalias associadas e grave insuficiência cardíaca no pré-operatório. Todos os sobreviventes apresentam bons resultados tardios e nenhum caso de recoarctação foi observado. Nāo houve a presença de aneurisma no grupo de pacientes submetidos à aortoplastia com enxerto, provavelmente pelo uso de enxertos biológicos. Os autores tentam individualizar a operação, escolhendo a técnica mais apropriada para cada caso. Entretanto, sempre que possível, usam a aortoplastia com sublcávia em crianças com menos de cinco anos e anastomose término-terminal ou aortoplastia com énxerto em pacientes mais idosos.
\end{abstract}

DESCRITORES: coarctação da aorta, cirurgica.

\section{INTRODUÇÃO}

Decorridos 45 anos da primeira cirurgia para correção da coarctação dą aorta, persistem controvérsias relativas às técnicas cirúrgicas atualmente em uso. A ressecção com anastomose término-terminal foi a primeira operação utilizada ${ }^{3}$, mas a impraticabilidade desta técnica em coarctaçōes longas levou ao emprego de homoenxertos ${ }^{4} \mathrm{e}$, posteriormente, de enxertos plásticos tubulares ". Por outro lado, a alta incidência de recoarctação, após anastomose término-terminal na infância, serviu de estímulo para o desenvolvimento das técnicas de aortoplastia com enxerto ${ }^{14}$ e com artéria subclávia ${ }^{15}$.
Ao longo de nossa experiência com o tratamento cirúrgico da coarctação da aorta, temos utilizado todas essas técnicas. Uma análise crítica das mesmas e uma revisão dos nossos resultados constituem o motivo do presente trabalho.

\section{CASUISTICA E MÉTODOS}

Entre 1965 e 1990, 70 pacientes portadores de coarctação da aorta foram operados no Hospital das Clínicas da UFPE, Instituto de Doenças do Tórax do Recife e Instituto do Coração de Pernambuco. Sessenta

\footnotetext{
Trabalho realizado no Serviço de Cirurgia Torácica do Hospital das Clínicas da Universidade Federal de Pernambuco e no Instituto do Coração de Pernambuco. Recife, PE, Brasil.

Recebido para publicaçăo em 1: de agosto de 1989

- Do Hospital das Clínicas da Universidade Federal de Pernambuco e do Instituto do Coraçăo de Pernambuco.

*. Do Instituto do Coraçăo de Pernambuco.

Endereço para separatas: Carlos Roberto Moraes. Av. Portugal, 163. 50000 Recife, PE, Brasil.
} 
MORAES, C. R.; RODRIgueS, J. V.; GOMES, C. A.; TENORIO, E.; MORAES NETO, F.; SANTOS, C. L.; CAVALCANTI, I. L. - Coarctaçăo da aorta: resultados da cirurgia e análise crítica de diversas técnicas. Rev. Bras. Cir. Cardiovasc., 4(2): 168-172, 1989.

e nove tinham coarctação primária e um apresentava recoarctaçāo, tendo sido operado a primeira vez em outro hospital. Cinqüenta e um $(72,8 \%)$ eram do sexo masculino e $19(27,2 \%)$, do sexo feminino, variando a idade de 14 dias a 49 anos (média de 7,6 anos). Na Tabela 1 observa-se a distribuiçăo por grupos etários, Vinte e seis $(37,1 \%)$ pacientes estavam no primeiro ano de vida. Lesões cardíaca associadas foram observadas em 26 $(37,1 \%)$ dos casos (Tabela 2).

A aorta foi exposta através de toracotomia pósterolateral esquerda em todos os casos. Diversas técnicas foram utilizadas nesta série (Tabela 3 ). Em 30 pacientes, de todos os grupos etários (Tabela 4), realizou-se aortoplastia com enxerto. Na grande maioria dos casos, empregaram-se enxertos biológicos (Tabela 5). No paciente portador de recoarctação, havia um gradiente pós-aortoplastia de $30 \mathrm{mmHg}$. Neste caso, associou-se um bypass com tubo de Goretex de $10 \mathrm{~mm}$ de diâmetro, entre a aorta proximal e distal à regiāo da coarctação.

Em 28 crianças, todas com idade inferior a cinco anos (Tabela 4), realizou-se aortoplastia com a artéria subclávia esquerda pela técnica clássica de WALDHAUSEN \& NAHRWOLD ${ }^{15}$. Em uma criança de dois anos de idade, a aortoplastia com artéria subclávia foi feita, preservando-se a circulaçăo para o membro superior esquerdo ${ }^{8}$.

Ressecção da coarctação seguida de anastomose término-terminal foi utilizada em nove pacientes de todas as faixas etárias (Tabela 4). Finalmente, em dois pacientes adultos, portadores de coarctaçāo muito longa, o restabelecimento da continuidade da aorta, após ressecção da coarctação, foi obtido pela interposição de um enxerto tubular de Dacron.

\section{RESULTADOS}

Seis $(8,5 \%)$ pacientes faleceram no pós-operatório imediato. Estes óbitos, resultados de insuficiência cardiaca em cinco casos e de coagulopatia em um, năo tiveram relação com o tipo de técnica utilizada, mas com a idade (inferior a um ano em cinco casos), presença de anomalias associadas e estado clínico (severa insuficiência cardíaca) no pré-operatório (Tabela 6).
Oito $(11,4 \%)$ outros pacientes apresentaram complicaçōes significativas, porém não fatais no pós-operatório imediato (Tabela 7), destacando-se dois casos de paraplegia. Em um desses pacientes, já anteriormente relatado ${ }^{10}$, a paraplegia ocorreu espontaneamente no segundo dia de pós-operatório após severa crise de hipertensão paradoxal. No outro, ocorreu após prolongado clam-

TABELA 1

DISTRIBUIÇÃO DAS IDADES 70 CASOS

\begin{tabular}{lcl}
\hline IDADE & $N \cdot$ & $\%$ \\
\hline $14 d-1$ ano & 26 & 37,1 \\
$1-5$ anos & 14 & 20,0 \\
$5-18$ anos & 16 & 22,9 \\
$18-49$ anos & 14 & 20,0 \\
\hline$d=$ dias & &
\end{tabular}

TABELA 2

ANOMALIAS ASSOCIADAS

\begin{tabular}{lcc}
\hline ANOMALIA & N: $=26$ & $37,1 \%$ \\
\hline PCA & 11 & $15,7 \%$ \\
CIV & 7 & $10,0 \%$ \\
EAo & 6 & $8,5 \%$ \\
TGA + CIV & 1 & $1,4 \%$ \\
Fibroelastose & 1 & $1,4 \%$
\end{tabular}

PCA = persistência do canal arterial; $\mathrm{CIV}$ = comunicação interventricular; EAo = estenose aórtica; TGA = transposição das grandes artérias.

TABELA 3

TIPOS DE OPERAÇÃO 70 CASOS

\begin{tabular}{lrr}
\hline TIPO & N. & \multicolumn{1}{c}{$\%$} \\
\hline AORTOPLASTIA COM ENXERTO & 30 & 42,8 \\
AORTOPLASTIA COM SUBCLÁVIA & 28 & 40,0 \\
AORTOPLASTIA COM SUBCLÁVIA (TELES) & 1 & 1,4 \\
ANASTOMOSE TÉRMINO-TERMINAL & 9 & 12,8 \\
ENXERTO TUBULAR & 2 & 2,8 \\
\hline
\end{tabular}

TABELA 4

RELAÇĀO IDADETIPO DE OPERAÇĀO

\begin{tabular}{|c|c|c|c|c|c|c|}
\hline$I D A D E$ & N:CASOS & $\begin{array}{l}\text { AORTOPLASTIA } \\
\text { COM ENXERTO }\end{array}$ & $\begin{array}{l}\text { AORTOPLASTIA } \\
\text { COM SUBCLAVIA }\end{array}$ & TELES & $\begin{array}{c}\text { ANASTOMOSE } \\
T-T\end{array}$ & $\begin{array}{l}\text { ENXERTO } \\
\text { TUBULAR }\end{array}$ \\
\hline$<1$ ano & 26 & 3 & 20 & - & 3 & - \\
\hline $1-5$ & 14 & 4 & 8 & 1 & 1 & - \\
\hline $5-18$ & 16 & 13 & - & - & 3 & - \\
\hline $18-49$ & 14 & 10 & - & - & 2 & 2 \\
\hline
\end{tabular}

$T \cdot T=$ término-terminal. 
MORAES, C. R.; RODRIGUES, J. V.; GOMES, C. A.; TENÓRIO, E.; MORAES NETO, F.; SANTOS, C. L.; CAVALCANTI, I. L. - Coarctaçāo da aorta: resultados da cirurgia e análise crítica de diversas técnicas. Rev. Bras. Cir. Cardiovasc., 4(2): 168-172, 1989.

\begin{tabular}{lr}
\multicolumn{2}{c}{ TABELA 5} \\
AORTOPLASTIA COM ENXERTO \\
3O CASOS
\end{tabular}

TABELA 6

MORTALIDADE IMEDIATA

\begin{tabular}{cccc}
\hline IDADE & $\begin{array}{c}\text { ANOMALIA } \\
\text { ASSOCIADA }\end{array}$ & $\begin{array}{c}\text { CLASSE } \\
\text { FUNCIONAL }\end{array}$ & $\begin{array}{c}\text { CAUSA DE } \\
\text { OBITO }\end{array}$ \\
\hline 22d & TGA + CIV & IV & Insuf. cardiaca \\
$37 \mathrm{~d}$ & CIV & IV & Insuf. cardiaca \\
$2 \mathrm{~m}$ & Fibroelastose & IV & Insuf. cardiaca \\
$11 \mathrm{~m}$ & CIV + EAO & III & Insuf. cardiaca \\
$3 \mathrm{~m}$ & CIV + PCA & IV & Insuf. cardiaca \\
$2 \mathrm{a}$ & - & II & Coagulopatia \\
\hline
\end{tabular}

$\mathbf{d}=$ dias; $\mathbf{m}=$ meses; $\mathbf{a}=$ anos; TGA = transposiçāo das grandes artérias; $\mathrm{CIV}=$ comunicação interventricular; $\mathrm{EAO}=$ estenose aórtica; PCA = persistência do canal arterial.

peamento da aorta para controle de sangramento no caso de recoarctação. Neste último doente, o quadro neurológico regrediu parcialmente.

Ocorreram dois $(2,8 \%)$ óbitos no pós-operatório tardio. Uma criança de sete anos teve morte súbita no terceiro mês, e uma outra faleceu no segundo ano de pós-operatório, durante correção cirúrgica de estenose sub-aórtica. Não se observaram, nos 62 sobreviventes, complicaçōes significativas, especialmente recoarctação e formação de aneurismas.

\section{DISCUSSĀO}

O tratamento cirúrgico da coarctação da aorta resultou numa mudança radical na história natural dessa máformação. Contudo, o aparecimento de uma série de complicaçōes tardias, decorrentes da própria doença ou inerentes às diversas técnicas utilizadas, tem tornado

TABELA 7

COMPLICACÓES NATO FATAIS

\begin{tabular}{lll}
\hline TIPO & N: & $\%$ \\
\hline HEMOTORAX & 4 & 5,7 \\
PARAPLEGIA & 2 & 2,8 \\
LESĀO RECURRENTE & 1 & 1,4 \\
DOR ABDOMINAL + MELENA & 1 & 1,4 \\
\hline
\end{tabular}

difícil uma avaliação dos resultados desse tipo de cirurgia a longo prazo.

Após a descrição original de CRAFOORD \& NY$\mathrm{LIN}^{3}$, a ressecção com anastomose término-terminal tornou-se a operaçāo padrāo para o tratamento cirúrgico da coarctação. Os resultados tardios desta técnica em pacientes com mais de um ano de idade são excelentes. Essa operação tem a vantagem de ressecar todo o tecido ductal e permitir o crescimento uniforme da aorta. No entanto, exige extensa dissecção, ligadura de várias artérias intercostais e, se a anastomose ficar sob tensão, o risco de sangramento é significativo. Por outro lado, a anastomose término-terminal năo pode ser realizada em coarctaçōes longas e, nesta situação, se o crescimento da aorta não entre em consideração, como em dois pacientes adultos de nossa série, a interposição de um enxerto tubular de Dacron é a operação de escolha com excelentes resultados. O grande problema da anastomose término-terminal, entretanto, relaciona-se à alta incidência de reestenose quando essa técnica é empregada no primeiro ano de vida ${ }^{7,13}$.

A aortoplastia com enxerto, originalmente descrita por VOSSCHULTE ${ }^{14}$, teoricamente tem várias vantagens sobre a anastomose término-terminal: exige menor dissecção da aorta e período mais curto de campleamento, determina pequena tensão na linha de sutura, o controle de sangramento é fácil, pois toda a linha de sutura é anterior e pode ser usada em qualquer tipo de coarctaçāo. Esta operação foi largamente utilizada em vários Serviços, incluindo o nosso ${ }^{9}$, até o aparecimento de relatos descrevendo a formaçāo tardia de aneurismas. BERGDAHL \& LJUNGQVIST ${ }^{1}$ descreveram essa complicação em 1980 e sua incidência em diversas séries tem variado de 5 a $38 \% 2,5,7$. A patogênese desses aneurismas, que quase sempre se formam na parede posterior da aorta, oposta ao enxerto de $\mathrm{Da}$ cron, não está esclarecida. Admite-se que a ressecção da membrana intema existente na zona da coarctação poderia contribuir para a formação desses aneurismas ${ }^{5}$. Por outro lado, sendo o enxerto de Dacron duro e inelástico, seria transmitida uma tensăo adicional à parede posterior da aorta que então se dilataria ${ }^{6}$. Esta complicação não tem sido observada em nossa série de 30 casos submetidos à aortoplastia, talvez pelo fato de termos utilizado enxertos flexíveis e elásticos na maioria dos doentes.

A aortoplastia com subclávia, introduzida por WALDHASEN \& NAHRWOLD ${ }^{14}$, em 1966, possui todas as vantagens teóricas das aortoplastias com enxerto e a vantagem adicional do enxerto ser autógeno e flexível. O potencial de crescimento da aorta em neonatos submetidos a este método foi demonstrado por MOULTON et alii $^{12}$. A incidência relatada de recoarctação e de outras complicaçōes com o emprego dessa técnica em crianças é muito baixa ${ }^{7}$, tornando-a operação de preferência da maioria dos cirurgiōes ${ }^{6}$. Utilizamos esse procedimento em 28 crianças com menos de cinco anos, obtendo excelentes resultados. 
MORAES, C. R.; RODRIGUES, J. V.; GOMES, C. A.; TENORIO, E.; MORAES NETO, F.; SANTOS, C. L.; CAVALCANTI, I. L. - Coarctaçăo da aorta: resultados da cirurgia e análise crítica de diversas técnicas. Rev. Bras. Cir. Cardiovasc., 4(2): 168-172, 1989.

Recentemente, MENDONÇA et alii ${ }^{8}$ propuseram uma nova técnica de aortoplastia com a artéria subclávia na qual a circulação para o membro superior esquerdo é mantida. Esta operação, realizada por nós em uma criança, é bastante engenhosa, mas, em nossa opinião, sua utilização deve ficar restrita aos casos de coarctação curta e próxima à emergência da artéria subclávia esquerda, evitando-se tensão na linha de sutura.

Atualmente, em nosso Serviço, temos procurado realizar, sempre que possível, a aortoplastia com subclávia em crianças com menos de cinco anos, e enastomose término-terminal ou aortoplastia com enxerto em pacientes acima daquela idade. Não há, porém, uma conduta rígida, pois acreditamos que a correção da coarctação da aorta deve ser uma operação individualizada. Assim, o cirurgiāo deve estar preparado para utilizar a melhor técnica para cada caso, visando a obter bons resultados a longo prazo, uma vez que, como foram bem demonstrados em nossa experiência, os resultados imediatos da cirurgia estão menos relacionados à técnica utilizada e mais vinculados a outros fatores de risco, especialmente à idade, à associação de outras lesōes e ao estado clínico pré-operatório.

MORAES, C. R.; RODRIgUeS, J. V.; GOMES, C. A.; TENÓRIO, E.; MORAES, NETO, F.; SANTOS, C. L.; CAVALCANTI, I. L. - Coarctation of the aorta: surgical results and critical analysis of several techniques. Rev. Bras. Cir. Cardiovasc., 4(2): 168-172, 1989.

ABSTRACT: Seventy patients ranging in age from 14 days to 49 years (mean 7.5 yrs.) were submitted to surgical repair of coarctation of the aorta. Twenty-six $(37.1 \%)$ were in the first year of life. Surgical techniques performed included patch aortoplasty in 30 cases, subclavian flap angioplasty in 28, resection and end-to-end anastomosis in 9, resection and interposition of a Dacron tube graft in 2 and subclavian aortoplasty with preservation of arterial blood flow to the left arm in 1 . There were $6(8.5 \%)$ early and $2(2.8 \%)$ late deaths, not related to the type of repair. Early mortality was clearly related to young age, associated anomalies and severe pre-operative heart failure. All surviving patients present good long-term results and no instance of recoarctation has occurred. No late aneurysm formation was seen in the group of patients submitted to patch aortoplasty probably due to the use of biological patchs in the majority of cases. At present, the authors try to individualize the operation, performing the more suitable technique for each case. Therefore, it is tried to use sublavian flap aortoplasty as much as possible in children below the age of 5 and end-to-end anastomosis or patch aortoplasty in older patients.

DESCRIPTORS: coarctation of the aorta, surgery.

\section{REFERÊNCIAS BIBLIOGRÁFICAS}

1 BERGDAHL, L. \& LJUNGQVIST, A. - Long-term results of coarctation of the aorta by patch grafting. J. Thorac. Cardiovasc. Surg., 80: 177-181, 1980.

2 CLARKSON, P. M.; BRANDT, P. W. T.; BARRATTBOYES, B. G. - Prosthetic repair of coarctation of the aorta with particular reference to Dacron patch grafts and late aneurysm formation. Am. J. Cardiol., 56: 342-346, 1985.

3 CRAFOORD, C. \& NYLIN, G. - Congenital coarctation of the aorta and its surgical treatment. J. Thorac. Surg., 14: 347-361, 1945.

4 GROSS, R. E. - Treatment of certain aortic coarctations by homologous grafts. Ann. Surg., 134: 753-750, 1951.

5 HEHRLEIN, F. W.; MULCH, J.: RAUTENBURG, H. W.; SCHLEPPER, M.; SCHELD, H. M. - Incidence and pathogenesis of late aneurysms after patch graft aortoplasty for coarctation. J. Thorac. Cardiovas. Surg., 92: 226-230, 1986.

6 KIRKLIN, J. W. \& BARRATT-BOYES, B. G. (eds.) - Cardiac surgery. New York, John Wiley \& Sons, Inc., 1986. p. $1036-1070$,

7 KRON, I. L.; FLANAGAN, T. L.; RHEUBAN, K. S.; CARPENTER, M. A.; GUTGSELL Jr., H. P.; BLACKBOURNE, L. H.; NOLAN, S. P. - Incidence and risk of reintervention after coarctation repair. Ann. Thorac. Surg., 49: 920-926, 1990.

8 MENDONÇA, J. T.; CARVALHO, M. R.; COSTA, R. K.; FRANCO FILHO, E. - Coarctation of the aorta: a new surgical technique. J. Thorac. Cardiovasc. Surg., 90: 445-447, 1985.

9 MORAES, C. R.; ARRUDA, M.; LAGRECA, J. R. - Tratamento cirúrgico da coarctação da aorta por aortoplastia. Arq. Bras. Cardiol., 28: 1971-1974, 1975. 
MORAES, C. R.; RODRIgUeS, J. V.; GOMES, C. A.; TENÓRIO, E.; MORAES NETO, F.; SANTOS, C. L.; CAVALCANTI, I. L. - Coarctaçăo da aorta: resultados da cirurgia e análise crítica de diversas técnicas. Rev. Bras. Cirr. Cardiovasc., 4(2): 168-172, 1989.

10 MORAES, C. R.; RODRIGUES, J. V.; LIMA, R. C.; ESCOBAR, M.; ARRUDA, M. B.; CAVALCANTI, I.; VICTOR, E. G. - Spontaneous development of paraplegia after repair of coarctation of the aorta. J. Thorac. Cardiovasc. Surg., 86: 942-943, 1985.

11 MORRIS, G. C.; COOLEY, D. A.: DeBAKEY, M. E.; CRAWFORD, E. S. - Coarctation of the aorta with particular emphasis upon improved techniques of surgical repair. J. Thorac. Cardiovasc. Surg., 40: 705-708, 1960.

12 MOULTON, A. L.; BRENNER, J. I.; ROBERTS, G.; TAVARES, S.; ALI, S.; NORDEMBERT, A.; BURNS, J. E.; RINGEL, R.; BERMAN, M. A. - Subclavian flap repair of coarctation of the aorta in neonates: realization of growth potencial. J. Thorac. Cardiovasc. Surg., 87: 220-235, 1984

13 SADE, R. M.; TAYLOR, A. B.; CHARIKER, E. P. - Aortoplasty compared with resection for coarctation of the aorta in young children. Ann. Thorac. Surg., 28: 346-353, 1979.

14 VOSSCHULTE, K. - Surgical correction of coarctation of the aorta by an isthmusplastic operation. Thorax, 16: $338-345,1961$

15 WALDHAUSEN, J. A. \& NAHRWOLD, D. L. - Repair of coarctation of the aorta with a subclavian flap. $J$. Thorac. Cardiovasc. Surg., 51: 532-533, 1966. 